\title{
Sessão de exercícios com o Nintendo Wii: comportamento das variáveis hemodinâmicas, gasto calórico e sensação subjetiva de esforço
}

\section{Exercise session with the Nintendo Wii: behavior of hemodynamic variables, caloric expense and feel exertion}

\author{
Raquel Cougo Alves, Thaís Becker Ventura, Silvana Corrêa Matheus, Diego \\ Rodrigo Both, Mauri Schwanck Behenck
}

http://dx.doi.org/10.11606/issn.2238-6149.v28i1p110-114

Alves RC, Ventura TB, Matheus SC, Both DR, Behenck MS.
Sessão de exercícios com o Nintendo Wii: comportamento das
variáveis hemodinâmicas, gasto calórico e sensação subjetiva de
esforço. Rev Ter Ocup Univ São Paulo. 2017 jan.-abr.;28(1):110-4.

RESUMO: Este estudo analisou o comportamento das variáveis hemodinâmicas, sensação subjetiva de esforço e gasto calórico durante uma sessão de exercícios físicos com o uso do Nintendo Wii Fit ${ }^{\circledR}$ de adultos jovens. Participaram sessenta voluntários ( 35 homens; 25 mulheres) com idade de $22,8 \pm 3,4$ anos (homens) e 21,5 2,6 anos (mulheres). A sessão de exercícios teve duração de 45 minutos e foi composta por nove jogos, através do Nintendo Wii Fitß. As variáveis hemodinâmicas e massa corporal foram coletadas em períodos de repouso, recuperação e ao término de cada jogo. $\mathrm{Na}$ análise estatística foram empregados: o teste de Shapiro-Wilk; Análise Descritiva e a Análise de Variância One Way (ANOVA); e o Post Hoc de Tukey, adotando como nível de significância estatística de $p<0,05$. Os resultados mostraram que a sessão de exercício produziu alterações cardiovasculares agudas e gasto calórico. Os dados obtidos neste estudo sugerem que uma sessão de 45 minutos de exercícios físicos promovidas pelo Wii Fit ${ }^{\circledR}$ pode ser utilizada como um modo eficaz de atividade física para melhorar a saúde.

DESCRITORES: Exercício; Homens; Mulheres; Adulto jovem; Jogos de vídeo.
Alves RC, Ventura TB, Matheus SC, Both DR, Behenck MS. Exercise session with the Nintendo Wii: behavior of hemodynamic variables, caloric expense and feel exertion. Rev Ter Ocup Univ São Paulo. 2017 Jan.-Apr.;28(1):110-4.

\begin{abstract}
This study analized the behavior of the hemodynamic variables, subjective sensation of effort and energy expenditure during an exercise session physicists using the Nintendo Wii Fit ${ }^{\circledR}$ of young adults. Sixty volunteers (35 men; 25 womens) were recruited from the university population, aged $22.8 \pm 3.4$ years (men) and $21.5 \pm 2.6$ years (women). The exercise session lasted 45 minutes and was composed of nine games, through the Nintendo Wii Fit ${ }^{\circledR}$. The hemodynamic variables were collected in periods of rest, recovery and the end of each game, along with the subjective sensation of effort and the body mass. In the Statistical analysis were employed: the Shapiro-Wilk test; Descriptive Analysis and Analysis of Variance One Way (ANOVA); and the post hoc Tukey. All analyses were conducted in SPSS, version 14.0 , with statistical significance set at $p<0.05$. Findings showed that the exercise session produced acute cardiovascular changes and caloric expenditure as recommended by the American College of Sport Medicine. Data from this study suggest that a session of 45 minute of the physical exercises fostered by Wii Fit $\AA$ can be used as an effective mode of physical activity to improve health.
\end{abstract}

KEYWORDS: Exercise; Men; Women; Young adult; Video games.

\footnotetext{
Este artigo foi resultado de Trabalho de Conclusão de Conclusão do Curso de Educação Física Bacharelado, da Universidade Federal de Santa Maria sendo vinculado ao Projeto Núcleo de Treinamento Físico com Realidade Virtual, sob registro no Gabinete de Projetos da instituição mencionada $n^{0}$ 032757. Coordenado pela Prof ${ }^{a} \operatorname{Dr}^{a}$ Silvana Corrêa Matheus.

1. Universidade Federal de Santa Maria. e-mails: raquel-ndn@hotmail.com, thais.becker.v@hotmail.com, silvanamatheus@gmail.com, diegoboth@yahoo.com.br, mbehenck@gmail.com

Endereço para correspondência: Raquel Cougo Alves. Rua Bento Gonçalves, 220 esquerda, bairro Centro, Bagé, RS. CEP: $96400-200$. E-mail: raquel-ndn@hotmail.com
} 


\section{INTRODUÇÃO}

A atividade física configura-se hoje como uma ferramenta importante no auxílio aos indivíduos para enfrentar as adversidades do cotidiano da melhor maneira. Sabe-se que a prática de exercício físico regular exerce influência positiva sobre parâmetros fisiológicos, metabólicos e psicológicos, proporcionando um menor risco de desenvolver doenças crônicas e uma menor possibilidade de morte prematura ${ }^{1-3}$.

Até um tempo atrás exercícios físicos não eram associados a videogames, pois a maioria desses jogos limitava a interação do jogador com o jogo através de um controle manual. Em 2006 vários jogos foram lançados, dentre eles o console Wii da Nintendo ${ }^{\circledR}$, que viabiliza a interação do usuário com o jogo através de situações similares a realidade. Neste sentido, estudos têm sido desenvolvidos acerca da utilização do Nintendo Wii®, como em aulas de educação física ${ }^{4}$, trabalhos com idosos ${ }^{5}$, entre outros. Porém, são poucos os estudos científicos acerca das respostas cardiovasculares agudas e o dispêndio energético durante a prática de exercícios realizados com o Nintendo Wii®.

Como a prática de atividade física pode ser um fator essencial para a melhora ou manutenção da qualidade de vida, a utilização do Nintendo Wii ${ }^{\circledR}$ com este propósito, torna-se uma forma alternativa de realizar exercícios físicos aliados à diversão. Sendo assim, o objetivo deste trabalho é analisar o comportamento das variáveis frequência cardíaca (FC), pressão arterial (PA), duplo produto (DP), sensação subjetiva de esforço (SSE) e gasto calórico (GCal), durante uma sessão de exercícios físicos com o uso do Nintendo Wii Fit ${ }^{\circledR}$ de homens e mulheres, saudáveis, com idade entre 18 e 30 anos.

\section{MÉTODOS}

Este trabalho foi realizado no Núcleo de Treinamento Físico com Realidade Virtual do Laboratório de Cineantropometria, no período entre os meses de julho a novembro de dois mil e treze em uma sala de $2,10 \mathrm{~m}$ de largura e de $3,10 \mathrm{~m}$ de comprimento equipada com 1 Nitendo Wii®, 1 Balance Board (acessório Wii), jogos eletrônicos e televisão LED colorida. Foram investigados 60 universitários, voluntários sendo 35 homens $(22,8 \pm 3,4$ anos) e 25 mulheres (21,5 2,6 anos). Utilizaram-se como critérios de inclusão: estar na faixa etária citada acima e assinar o Termo de Consentimento Livre e Esclarecido (TCLE). A pesquisa se caracteriza como quase experimental ${ }^{6}$, pois analisou o comportamento das variáveis hemodinâmicas, SSE e GCal durante uma sessão de treinamento físico (STF) com o uso do Nintendo Wii Fit ${ }^{\circledR}$ não apresentando aleatoriedade na seleção dos indivíduos.

No primeiro dia agendado os indivíduos passaram por avaliação antropométrica através de medidas de $\mathrm{MC}$ (balança Marte ${ }^{\circledR}$; resolução $0,1 \mathrm{~kg}$; capacidade de $180 \mathrm{~kg}$ ), estatura (estadiômetro fixo Cardiomed $^{\circledR}$ com resolução de $0,1 \mathrm{~cm}$ ) e circunferência do braço (relaxado) para a correção da PA. A MC e a estatura foram coletadas de acordo com os procedimentos descritos por Stewart et al. ${ }^{7}$. Ainda neste dia receberam as recomendações precedentes a STF referentes à ingestão de cafeína, bebida alcoólica, alimentos, uso do cigarro e prática de exercícios físicos.

A STF foi realizada em um segundo dia e teve duração de 45 minutos sendo composta por nove jogos representando exercícios aeróbicos, de força e de yoga com o uso do Nintendo Wii Fit $\mathbb{R}$. Antes de iniciar a STF foram verificadas a FC (frequencímetro Polar ${ }^{\circledR}$ - FS1) e a PA (esfigmomanômetro aneróide Solidor ${ }^{\circledR}$ e estetoscópio $\mathrm{Bic}^{\circledR}$ ), através do método auscultatório, utilizando-se um manguito padrão $(13 \times 24 \mathrm{~cm})$ após permanecer cinco minutos sentado (coleta de repouso). Os valores de PA obtidos foram corrigidos de acordo coma circunferência do braço dos sujeitos ${ }^{8}$. Ambas foram coletadas seguindo os procedimentos descritos nas VI Diretrizes Brasileiras de Hipertensão9. Logo após, os avaliados fizeram o reconhecimento da plataforma a partir das instruções sobre o funcionamento da mesma. Em seguida, iniciava-se a STF, constituída por jogos das categorias: Aeróbicos (25 min): Basic Step; Hula Hop; Island Cyclinge e Free Run. Força (15 min): Rowing Squat; Lunge; e Plank. e Yoga (5 min): Warrior e Palm Tree. Ao final de cada jogo foram verificadas a FC, a PA, DP e SSE ${ }^{10}$. E ao final da STF foram medidas a FC, PA e DP durante a recuperação (5 min). O DP foi calculado multiplicando-se a PA sistólica (PAS) pela $\mathrm{FC}^{11}$. Para o resultado do GCal, utilizou-se a informação dos equivalentes metabólicos (MET's) de cada atividade fornecida pelo próprio jogo e a seguinte fórmula: $\mathrm{GCal}(\mathrm{kcal} / \mathrm{min})=($ MET $\times 3,5 \times \mathrm{MC}$ em kg $) / 200^{12}$.

O estudo foi aprovado pelo Comitê de Ética em Pesquisa com seres humanos (CAAE $n^{\circ}$ 31095214.0.0000.5346) e os voluntários assinaram o Termo de Consentimento Livre e Esclarecido (TCLE), de acordo com a resolução 196/96 do Conselho Nacional de Saúde.

\section{Análise Estatística}

Utilizou-se o teste de Kolmogorov-Smirnov (normalidade dos dados), a análise descritiva, a Análise de Variância One Way (ANOVA) (diferença das médias 
Alves RC, et al. Sessão de exercícios com o Nintendo Wii. Rev Ter Ocup Univ São Paulo. 2017 jan.-abr.;28(1):110-4.

dos diferentes momentos de coleta) e o post Hoc de Tukey (identificar as variáveis que diferiram entre os momentos de análise), por intermédio do software SPSS versão 14.0, adotando como nível de significância 5\%.

\section{RESULTADOS}

Na Tabela 1 são apresentadas as médias dos dados durante os diferentes momentos da STF dos 60 sujeitos investigados. Ao analisar as variáveis hemodinâmicas durante o repouso e a STF constata-se que os maiores valores de PAS, FC e DP foram encontrados no jogo Free Run (categoria aeróbicos), sendo significativamente superior ao jogo Hula Hop e demais jogos. Já a PAD foi maior no jogo Island Cycling (categoria aeróbicos), sendo significativamente superior aos jogos Free Run, Lunge e Plank, não diferindo dos demais jogos e da recuperação. Ao analisar a SSE as maiores médias foram encontradas no jogo Plank, seguido do Free Run, não havendo diferença significativa dos resultados desta variável comparando os dois jogos entre si e estes com o Lunge, no entanto, os resultados do Plank foram significativamente superiores aos obtidos nos demais jogos. Em relação ao GCal observou-se que os maiores resultados foram obtidos nos jogos Free Run e Hula Hop os quais, além de diferirem entre si, diferiram significativamente dos dados obtidos nos demais jogos.

Tabela 1 - Resultados das variáveis hemodinâmicas, SSE e Gasto calórico obtidos durante os diferentes momentos de coleta

\begin{tabular}{|c|c|c|c|c|c|c|c|c|c|c|c|}
\hline \multirow{2}{*}{$\mathrm{VA}$} & \multicolumn{6}{|c|}{ Aeróbicos } & \multicolumn{3}{|l|}{ Força } & \multicolumn{2}{|l|}{ Yoga } \\
\hline & *Rep & *Bas & *Hula & *Islan & *Free & *Squat & *Lung & *Plan & *War & *Palm & ${ }^{*} \operatorname{Rec}$ \\
\hline PS & $\begin{array}{l}117,5 \\
(9,1) \\
\text { a }\end{array}$ & $\begin{array}{l}126,5 \\
(11,3) \\
\text { b }\end{array}$ & $\begin{array}{l}141,9 \\
(18,8) \\
c\end{array}$ & $\begin{array}{l}135,4 \\
(14,3) \\
\mathrm{cd}\end{array}$ & $\begin{array}{l}150,5 \\
(19,7) \\
\mathrm{e}\end{array}$ & $\begin{array}{l}132,5 \\
(12,3) \\
\mathrm{f}\end{array}$ & $\begin{array}{l}132,7 \\
(16) \\
\text { fg }\end{array}$ & $\begin{array}{l}130,6 \\
(16,4) \\
\text { fgh }\end{array}$ & $\begin{array}{l}124,0 \\
(13,2) \\
\text { abfghi }\end{array}$ & $\begin{array}{l}119,0 \\
(14,3) \\
\text { abij }\end{array}$ & $\begin{array}{l}116,0 \\
(8,1) \\
\text { aijl }\end{array}$ \\
\hline PD & $\begin{array}{l}68,4 \\
(7,8) \\
a\end{array}$ & $\begin{array}{l}69,6 \\
(10,1) \\
\text { ab }\end{array}$ & $\begin{array}{l}68,5 \\
(8,9) \\
a b c\end{array}$ & $\begin{array}{l}71,6 \\
(9,4) \\
\text { abcd }\end{array}$ & $\begin{array}{l}64,5 \\
(12,6) \\
\text { abce }\end{array}$ & $\begin{array}{l}67,2 \\
(8,2) \\
\text { abcdef }\end{array}$ & $\begin{array}{l}63,1 \\
(9,7) \\
\text { abcefg }\end{array}$ & $\begin{array}{l}64,0 \\
(12,6) \\
\text { abcefgh }\end{array}$ & $\begin{array}{l}70,6 \\
(10) \\
\text { abcdefghi }\end{array}$ & $\begin{array}{l}69,7 \\
(8,2) \\
\text { abcdefghij }\end{array}$ & $\begin{array}{l}69,2 \\
(8,6) \\
\text { abcdefghij }\end{array}$ \\
\hline $\mathrm{FC}$ & $\begin{array}{l}68,7 \\
(11,1) \\
a\end{array}$ & $\begin{array}{l}101,1 \\
(14,4) \\
\text { b }\end{array}$ & $\begin{array}{l}146,8 \\
(24,2) \\
c\end{array}$ & $\begin{array}{l}123,8 \\
(20,5) \\
d\end{array}$ & $\begin{array}{l}167,5 \\
(18,8) \\
\mathrm{e}\end{array}$ & $\begin{array}{l}133,3 \\
(18,4) \\
\text { cf }\end{array}$ & $\begin{array}{l}142,8 \\
(17,1) \\
\text { cfg }\end{array}$ & $\begin{array}{l}132,2 \\
(22,0) \\
\text { cfgh }\end{array}$ & $\begin{array}{l}119,5 \\
(18,5) \\
\mathrm{i}\end{array}$ & $\begin{array}{l}110,0 \\
(17,5) \\
\text { bij }\end{array}$ & $\begin{array}{l}83,4 \\
(13,4) \\
1\end{array}$ \\
\hline DP & $\begin{array}{l}8083 \\
(1140,5) \\
\mathrm{a}\end{array}$ & $\begin{array}{l}12790 \\
(2076) \\
b\end{array}$ & $\begin{array}{l}20994 \\
(5172) \\
c\end{array}$ & $\begin{array}{l}16738 \\
(3216) \\
d\end{array}$ & $\begin{array}{l}25249 \\
(4356) \\
\mathrm{e}\end{array}$ & $\begin{array}{l}17645 \\
(2767) \\
f\end{array}$ & $\begin{array}{l}18961 \\
(3223) \\
\mathrm{fg}\end{array}$ & $\begin{array}{l}17227 \\
(3359) \\
\text { fgh }\end{array}$ & $\begin{array}{l}14808 \\
(2466) \\
\mathrm{i}\end{array}$ & $\begin{array}{l}1306 \\
(2050) \\
\text { bij }\end{array}$ & $\begin{array}{l}9716 \\
(1547) \\
\text { al }\end{array}$ \\
\hline SSE & ------ & $\begin{array}{l}7,2 \\
(1,3) \\
b\end{array}$ & $\begin{array}{l}11,9 \\
(2,3) \\
c\end{array}$ & $\begin{array}{l}11,7 \\
(2,6) \\
d\end{array}$ & $\begin{array}{l}13,6 \\
(2,8) \\
\mathrm{e}\end{array}$ & $\begin{array}{l}10,6 \\
(2,5) \\
f\end{array}$ & $\begin{array}{l}12,9 \\
(2,7) \\
\text { eg }\end{array}$ & $\begin{array}{l}13,7 \\
(2,8) \\
\text { egh }\end{array}$ & $\begin{array}{l}8,6 \\
(2,5) \\
\text { bi }\end{array}$ & $\begin{array}{l}8,3 \\
(2,0) \\
\text { bij }\end{array}$ & ------- \\
\hline GCal & $\begin{array}{l}------- \\
----\end{array}$ & $\begin{array}{l}3,9 \\
(0,8) \\
\mathrm{b}\end{array}$ & $\begin{array}{l}5,2 \\
(1,0) \\
c\end{array}$ & $\begin{array}{l}3,3 \\
(0,6) \\
d\end{array}$ & $\begin{array}{l}6,6 \\
(1,3) \\
\mathrm{e}\end{array}$ & & $\begin{array}{l}4,6 \\
(0,9) \\
\mathrm{f}\end{array}$ & $\begin{array}{l}3,9 \\
(0,8) \\
b g\end{array}$ & $\begin{array}{l}2,9 \\
(0,6) \\
h\end{array}$ & $\begin{array}{l}2,6 \\
(0,5) \\
\mathrm{hi}\end{array}$ & $\begin{array}{l}2,6 \\
(0,5)^{\text {hij }}\end{array}$ \\
\hline
\end{tabular}

Os dados apresentados na tabela são referentes às médias (desvio padrão). $\mathrm{FAS}=$ fases da sessão; VA= variáveis. *Momentos de análise. REP= repouso; $\mathrm{BAS}=$ basic step; HULA = hua hop; INSLAN= insland cycling; FREE= free run; SQUAT= rowing squat; $L U N G=$ lunge; $P L A N=$ plank; $P A L M=$ palm tree; $R E C=$ recuperação. VAR= variáveis; $\mathrm{PS}=$ pressão arterial sistólica; $\mathrm{PD}=$ pressão arterial diastólica; $\mathrm{FC}=$ frequência cardíaca; $\mathrm{DP}=$ duplo produto; $\mathrm{SSE}=$ Sensação Subjetiva de Esforço e Gcal= Gasto Calórico. Letras diferentes representam diferença estatisticamente significativa entre os diferentes momentos da STF $\mathrm{p}<0,05$.

\section{DISCUSSÃO}

Para obtenção da saúde é recomendado uma variação alvo de 150 a $400 \mathrm{Kcal} /$ dia de GCal através da atividade e/ou exercício físico ${ }^{13}$. Ao analisar esta variável durante a STF identificou-se que se encontra dentro destas recomendações, proporcionando um GCal de 190,2 kcal durante os 45 min. de realização. Dados semelhantes a estes foram obtidos em um estudo ${ }^{14}$ que analisou o GCal e a FC de 13 adultos jovens, que praticaram três jogos utilizando realidade virtual, totalizando 30 minutos. Os resultados apontaram um GCal total de 226,07 $\pm 48,68 \mathrm{kcal}$.

Em relação às respostas cardiovasculares ao exercício físico, sabe-se que, tanto a PAS quanto a FC aumentam em decorrência da execução de exercícios estáticos, bem como, de dinâmicos, devido a ativação dos quimiorreceptores e aumento da atividade simpática atribuídos aos exercícios estáticos; e a ativação do comando central, de mecanorrecptores musculares, além do aumento da atividade simpática, atribuídos aos exercícios dinâmicos ${ }^{15}$. Quanto a PAD, a tendência é a 
manutenção dos valores com pequenas oscilações ${ }^{15}$. Os resultados encontrados no presente trabalho corroboram com os achados da literatura, haja vista que, tanto nos jogos caracterizados como estáticos quanto nos dinâmicos a PAS e a FC do grupo investigado apresentaram valores significativamente superiores aos obtidos em repouso. Já a PAD, em ambos os exercícios, apresentou pequenas oscilações em relação aos valores de repouso, porém sem nenhuma significância estatística.

Douris et al. ${ }^{16} \mathrm{em}$ seu estudo envolvendo adultos jovens sedentários e saudáveis compararam as respostas fisiológicas e psicológicas destes, obtidas no exercício aeróbio em esteira, com as obtidas com o jogo Free Run

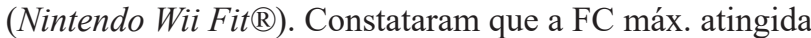
com o Wii Fit $(142,4 \pm 20,5 \mathrm{bpm})$ foi significativamente maior em comparação com exercício na esteira $(123,2$ $\pm 13,7 \mathrm{bpm}$ ). O mesmo aconteceu com a SSE (Free Run $=12,7 \pm 3,0$ vs Esteira $=10,1 \pm 3,3$ ). Tais resultados se assemelham aos encontrados neste trabalho, visto que, as maiores médias para a FC e DP foram encontradas no jogo Free Run, assim como, a média da SSE que foi a maior entre todos os jogos juntamente com o exercício Plank.

Souza et al. ${ }^{17}$ analisaram as respostas agudas da FC, PA e DP de universitários saudáveis,ao realizarem 25 arremessos de basquete utilizando o software Wii Sports Resort da Nintendo, em duas situações: sentados e saltando verticalmente. Os autores constataram alteração na FC nas duas situações (FC sentado $=51,62 \%$ e FC salto $75,34 \%$ da FC Max). No entanto, observaram que a resposta foi maior para a segunda situação de análise e justificam a ocorrência de tal resposta devido ao maior recrutamento muscular ao executar o salto vertical. O mesmo nota-se nos resultados encontrados na sessão de exercícios propostos no presente estudo, onde os maiores valores médios para a FC, PAS e DP, foram encontrados no jogo Free Run, que, ao simular uma corrida promoveu maior recrutamento muscular quando comparado aos outros exercícios.

\section{REFERÊNCIAS}

1. Colberg SR. Physical activity: the forgotten tool for type 2 diabetes management. Front Endocrinol. 2012;3(70):1-6. doi: $10.3389 / 2012.00070$.

2. Pacheco DRR, Silva MJB, Alexandrino AMS, Torres RMT. Exercise-related quality of life in subjects with asthma: a systematic review. J Asthma. 2012;49(5):487-95. doi: 10.3109/02770903.2012.680636.
De acordo com a literatura, o DP tende a aumentar durante as atividades físicas, no entanto, o seu comportamento é dependente da natureza da solicitação ${ }^{18}$. Fato que justifica os valores inferiores constatados nos jogos de força (rowing squat $=17645,1 \pm 2767$ e lunge $=8961,1 \pm 3223,3)$ ao comparar com os encontrados em Simão et al. ${ }^{17}$ (agachamento horizontal $=25010 \pm 3253,5$; agachamento vertical $=28489 \pm 3934,1)$, pois neste estudo verificou-se a FC, a PA e o DP, durante exercícios contra-resistência e na SEF proposta não se fez uso de sobrecarga.

Hui et al. ${ }^{19}$ desenvolveram valores normativos de DP em diferentes situações: DP de repouso (DPRep) $=$ 7524; DP submáximo (DPSub) = 21218; e DP máximo $($ DPMáx $)=32798$, com um grupo de indivíduos clinicamente saudáveis e correlacionaram com as variáveis da aptidão física. Em conclusão afirmam que um DPMáx de 15000 ou inferior é um importante preditor de morte. Ao comparar tais resultados com os obtidos no presente estudo, constatou-se que a média do DPRep $(8083,0)$ está um pouco acima da média normatizada. Apenas no exercício Free Run $(25248,8)$ foi encontrado valor acima da média estipulada para o DPSub e nenhum exercício proposto produziu valores de DP que pudessem ser enquadrados na categoria de DPMáx. Cabe ressaltar que, o DP pode ser utilizado como parâmetro para controlar a intensidade e o risco de uma atividade física, pois a FC e a PA consideradas isoladamente, nem sempre podem garantir segurança ${ }^{20}$.

Com base nos resultados obtidos, concluise que uma STF estruturada de 45 minutos realizada com o Nintendo Wii Fit ${ }^{\circledR}$ é capaz de gerar alterações cardiovasculares de forma aguda e proporciona um GCal dentro do recomendado pelo ACSM. Sendo assim, infere-se que o uso desta tecnologia de forma estruturada, sistemática e regular, pode ser uma ferramenta alternativa para a melhora da aptidão física relacionada à saúde.

3. Stoller O, De Bruin ED, Knols RH, Hunt KJ. Effects of cardiovascular exercise early after stroke: systematic review and meta-analysis. BMC Neurol. 2012;12:45. doi: 10.1186/1471-2377-12-45.

4. Vaghetti CAO, Botelho SSC. Ambientes virtuais de aprendizagem na educação física: uma revisão sobre a utilização de exergames. Ciên Cogn. 2010;15(1):76-88. 
Alves RC, et al. Sessão de exercícios com o Nintendo Wii. Rev Ter Ocup Univ São Paulo. 2017 jan.-abr.;28(1):110-4.

Disponível em: http://cienciasecognicao.org/revista/index. $\mathrm{php} / \mathrm{cec} /$ article/view/292/162.

5. Portela FJR. Nintendo Wii ${ }^{\circledR}$ qual o impacto na $3^{\mathrm{a}}$ idade? Estudo clínico randomizado [dissertação]. Porto: Faculdade de Medicina da Universidade do Porto; 2010. Disponível em: https://repositorio-aberto.up.pt/bitstream/10216/55344/2/ FilipePortelaNintendoWiiQualoimpactona3IdadeEstudoClinicoRandomizadoTeseMestrado.pdf.

6. Thomas JR, Nelson JK, Silverman, SJ. Métodos de pesquisa em atividade física. Porto Alegre: Artmed; 2007.

7. Stewart A, Marfell-Jones M, Olds Timothy, Ridder R. International standards for anthropometric assessment. Australia: The International Society for the Advancement of Kinanthropometry, National Library of Australia; 2011.

8. Ferreira SRS, Bianchini IM, Glasenapp R, Nader EK, organizadores; Ministério da Saúde. Grupo Hospitalar Conceição. Gerência de Saúde Comunitária. Protocolo de hipertensão arterial sistêmica para a atenção primária em saúde. Porto Alegre: Hospital Nossa Senhora da Conceição S.A.; 2009. Disponível em: http://www2.ghc.com.br/ GepNet/publicacoes/protocolodehipertensao.pdf.

9. Sociedade Brasileira de Cardiologia. Sociedade Brasileira de Hipertensão. Sociedade Brasileira de Nefrologia. VI Diretrizes Brasileiras de Hipertensão. Arq Bras Cardiol. 2010;95(1):1-51. Disponível em: http://publicacoes.cardiol. br/consenso/2010/Diretriz_hipertensao_associados.pdf.

10. Borg GAV. Psychophysical bases of perceived exertion. Med Sci Sports Exerc. 1982;14(3):77-81. Disponível em: http://fcesoftware.com/images/15_Perceived_Exertion.pdf.

11. Fornitano LD e Godoy MF. Duplo produto elevado como preditor de ausência de coronariopatia obstrutiva de grau importante em pacientes com teste ergométrico positivo. Arq Bras Cardiol. 2006;86(2)138-44. Disponível em: http:// www.scielo.br/pdf/abc/v86n2/a10v86n2.pdf.

12. American College of Sports Medicine (ACSM). Diretrizes do ACSM para os testes de esforço e sua prescrição. Rio de Janeiro: Guanabara Koogan; 2007.

13. Garber $\mathrm{CE}$, Blissmer BD, Michael R, Franklin BA, Lamonte MJ, Lee I-Minmd, et al. American College of Sports Medicine quantity and quality of exercise for developing and maintaining cardiorespiratory, musculoskeletal, and neuromotor fitness in apparently healthy adults: guidance for prescribing exercise. Med Sci Sports Exerc. 2011;43(13):34-59. doi: 10.1249/ MSS.0b013e318213fefb.

14. Siegel SR, Haddock BL, Dubois AM, Wilkin LD. Active video/arcade games (exergaming) and energy expenditure in college students. Int J Exerc Sci 2009;2(3):165-74. Available from: http://digitalcommons.wku.edu/cgi/ viewcontent . cgi article $=1062 \&$ context $=$ ijes

15. Brum PC, Forjaz CLM, Tinucci T, Negrão CE. Adaptações agudas e crônicas do exercício físico no sistema cardiovascular. Rev Paul Educ Fís. 2004;18:2131. Disponível em: http://www.luzimarteixeira.com.br/ wp-content/uploads/2009/11/adaptacoes-musculares-aoexercicio-fisico1.pdf.

16. Douris PC, McDonald B, Vespi F, Kelley NC, Herman L. Comparison between Nintendo Wii Fit aerobics and traditional aerobic exercise in sedentary young adults. J Strength Cond Res. 2012;26(10):52-7. doi: 10.1519/ JSC.0b013e31822e5967

17. Souza RA, Cruz LG, Carvalho PS, Silva FF, Carvalho WRG. Respostas cardiovasculares agudas em ambiente virtualmente simulado pelo Nintendo Wii. Rev Bras Cineantropom Desempenho Hum. 2013;15(1):60-70. doi: 10.5007/1980-0037.2013v15n1p60.

18. Simão R, Polito MD, Lemos A. Comportamento do duploproduto em diferentes posições corporais nos exercícios contra-resistência. Fit Perf J. 2003;2(5):279-84. doi: 10.3900/fpj.2.5.279.

19. Hui SC, Jackson AS, Wier L.T. Development of normative values for resting and exercise rate pressure product. Med Sci Sports Exerc. 2000;32(8):1520-27. Disponível em: https://www.ncbi.nlm.nih.gov/pubmed/10949021.

20. Farinatti PTV, Assis BFCB. Estudo da Frequência Cardíaca, Pressão Arterial e Duplo-produto em exercícios contraresistência e aeróbio contínuo. Rev Bras Ativ Fís Saúde. 2000;5(2):5-16. doi: 10.12820/rbafs.v.21n5p388-399.

Recebido em: 19.09.15

Aceito em: 17.1.17 